\title{
Differences in caecal microbiota composition and Salmonella carriage between experimentally infected inbred lines of chickens
}

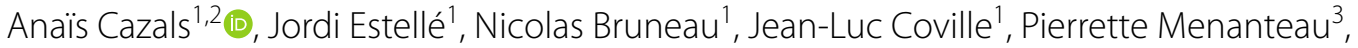 \\ Marie-Noëlle Rossignol', Deborah Jardet ', Claudia Bevilacqua' , Andrea Rau', Bertrand Bed'Hom', \\ Philippe Velge ${ }^{3}$ and Fanny Calenge ${ }^{1 *}$
}

\begin{abstract}
Background: Salmonella Enteritidis (SE) is one of the major causes of human foodborne intoxication resulting from consumption of contaminated poultry products. Genetic selection of animals that are more resistant to Salmonella carriage and modulation of the gut microbiota are two promising ways to decrease individual Salmonella carriage. The aims of this study were to identify the main genetic and microbial factors that control the level of Salmonella carriage in chickens (Gallus gallus) under controlled experimental conditions. Two-hundred and forty animals from the White Leghorn inbred lines $N$ and $\sigma_{1}$ were infected by SE at 7 days of age. After infection, animals were kept in isolators to reduce recontamination of birds by Salmonella. Caecal contents were sampled at 12 days post-infection and used for DNA extraction. Microbiota DNA was used to measure individual counts of SE by digital PCR and to determine the bacterial taxonomic composition, using a 16S rRNA gene high-throughput sequencing approach.
\end{abstract}

Results: Our results confirmed that the $\mathrm{N}$ line is more resistant to Salmonella carriage than the $\sigma_{1}$ line, and that intraline variability is higher for the 6 line. Furthermore, the $16 \mathrm{~S}$ analysis showed strong significant differences in microbiota taxonomic composition between the two lines. Among the 617 operational taxonomic units (OTU) observed, more than 390 were differentially abundant between the two lines. Furthermore, within the 61 line, we found a difference in the microbiota taxonomic composition between the high and low Salmonella carriers, with 39 differentially abundant OTU. Using metagenome functional prediction based on $16 \mathrm{~S}$ data, several metabolic pathways that are potentially associated to microbiota taxonomic differences (e.g. short chain fatty acids pathways) were identified between high and low carriers.

Conclusions: Overall, our findings demonstrate that the caecal microbiota composition differs between genetic lines of chickens. This could be one of the reasons why the investigated lines differed in Salmonella carriage levels under experimental infection conditions.

*Correspondence: fanny.calenge@inrae.fr

1 Université Paris-Saclay, INRAE, AgroParisTech, GABI, 78350 Jouy-en-Josas, France

Full list of author information is available at the end of the article

\section{Background}

Reducing Salmonella carriage in chicken flocks is important to ensure human food safety and enhance breeding viability. Indeed, consumption of contaminated raw poultry products can cause human food intoxications. Between 2014 and 2016, more than 4000 cases of original author(s) and the source, provide a link to the Creative Commons licence, and indicate if changes were made. The images or other third party material in this article are included in the article's Creative Commons licence, unless indicated otherwise in a credit line to the material. If material is not included in the article's Creative Commons licence and your intended use is not permitted by statutory regulation or exceeds the permitted use, you will need to obtain permission directly from the copyright holder. To view a copy of this licence, visit http://creativecommons.org/licenses/by/4.0/. The Creative Commons Public Domain Dedication waiver (http://creativeco mmons.org/publicdomain/zero/1.0/) applies to the data made available in this article, unless otherwise stated in a credit line to the data. 
human salmonellosis outbreaks due to the consumption of contaminated chicken meat and eggs were reported in Europe with more than 93\% due to the Enteritidis serovar [1]. To decrease the number of cases, hygiene control measures, detection of serovars of Salmonella, and culling of contaminated flocks or vaccination have been implemented on farms. Nevertheless, the economic impact of human salmonellosis is estimated at approximately 3 billion euros per year [1]. Thus, developing new strategies to improve the control of Salmonella propagation in poultry livestock is essential to meet this challenge.

In adult chickens, Salmonella enterica Enteritidis (SE) does not induce symptoms and can remain in the host for a long time [2]. Carrier animals excrete the bacteria in the environment, thus increasing their propagation and facilitating contamination of other chickens. Previous studies have identified several quantitative trait loci (QTL) associated with Salmonella carriage in the White Leghorn inbred lines $N$ and $66_{1}$ [3-5], which shows that differences in host genetics contribute to resistance to Salmonella carriage. However, the effects associated with these QTL are weak and do not allow direct application of marker-assisted selection for more resistant animals in commercial lines. The weak effects of these QTL might also result from biased quantification of Salmonella carriage in individual chickens, due to the recurrent recontamination of birds by Salmonella that are excreted by other birds within the timeframe of experimental infections. This recontamination probably leads to a homogenization of Salmonella carriage in chicken flocks. In fact, the use of isolators, which strongly reduces this recontamination, has led to much greater individual variation between birds in the experimental White Leghorn line PA12 [2], and has allowed the description of three categories of birds based on their level of shedding: super-, intermediate- and low-shedders. Through the use of grids on the floor and decontamination of faecal drops, isolators allow control of air purity and limit faecal-oral recontamination between birds.

In addition to the effect of host genetics, many studies have demonstrated the importance of the gut microbiota for the host's health, not only in poultry, but also in other livestock species and in humans. In humans, disruptions of the intestinal microbiota can lead to many kinds of diseases by altering the host's physiology and metabolism and triggering inflammation [6]. It is now clear that both the host and its intestinal microbiota contribute to the expression of many phenotypic traits of interest in livestock species [7]. In chickens, it is also well established that intestinal health is the result of complex functional interactions between intestinal microbes and host immunity [8]. In particular, the gut microbiota of adult chickens has been consistently identified as a protective factor to prevent colonisation of the intestine of young chicks by Salmonella sp. through a mechanism called competitive exclusion [911]. Although the primary site of Salmonella invasion in the host is the small intestine, the caecal microbiota plays a key role in susceptibility of the host to SE caecal colonization and on Salmonella shedding. The caecal microbiota composition before SE infection has been shown to be associated with the Salmonella super- or low-shedder phenotypes in the chicken lines $\mathrm{N}$ and $6_{1}$ [12]. And indeed, spraying adult microbiota onto young chicks or the use of probiotics are already effectively used in commercial flocks to reduce SE load [13-16]. Many studies have also assessed the effectiveness of nutritional strategies to reduce SE load by modulating the intestinal microbiota, which results in an improvement of the host immunity [17-20]. Such strategies include prebiotics or probiotics that boost production of beneficial metabolites, modulate host immunity, and improve function of the intestinal barrier [21].

To understand the underlying biological mechanisms explaining the reduction of SE load through nutritional strategies, and to identify the bacterial taxa realising the competitive exclusion of Salmonella, it is important to analyse the impact of Salmonella infection on host microbiota composition. By comparing infected and noninfected animals, several studies have identified Operational Taxonomic Units (OTU) signatures of Salmonella infection. For example in pigs, Argüello et al. [22] identified bacteria from the Clostridia class that could prevent Salmonella Typhimurium colonisation. In chicken, the Ruminococcaceae family, which is more abundant in noninfected animals at 4 days post-infection (dpi), could be a signature of Salmonella infection [23].

In conclusion, both host genetics and composition of the intestinal microbiota can explain differences in SE carriage in chickens. Host genetics and intestinal microbiota may also interact, since genetic studies of other phenotypes, such as digestive efficiency [24], body weight [25] or feather-pecking in laying hens [26], have shown that host genetics influences intestinal microbiota composition in chickens. In these studies, several QTL and single nucleotide polymorphisms (SNPs) that were associated with specific bacterial species and may explain the phenotype were identified, and significant heritabilities of the abundance of these bacteria were found.

In this study, we investigated the combined influence of host genetics and gut microbiota composition on caecal SE load. Using the $N$ and $6_{1}$ inbred chicken lines, we studied the impact of the host genetics on both Salmonella carriage and microbiota composition at the individual level, using the isolator model, which highly decreases 
the exchange of gut microbiota between animals [2]. Our aims were to:

(i) Assess the individual variability in Salmonella load and in caecal microbiota composition within each line;

(ii) Compare Salmonella load and caecal microbiota composition of the two $\mathrm{N}$ and $6_{1}$ lines, to investigate the existence of a genetic control of these two phenotypes; and

(iii) Identify putative microbial signatures of low/high Salmonella carriage within and between the two lines.

\section{Methods}

\section{Experimental design}

Two experiments (i.e. Experiments 1 and 2) were conducted 1 month apart (January and February 2017) using birds from the White Leghorn inbred lines $N$ and 6 . For each experiment (Fig. 1), chicks from the two lines (in total, $\mathrm{n}=120 ; 30$ males and 30 females from each line) were hatched together with free access to feed [for feed composition (see Additional file 1)] and water at the experimental unit PEAT (Pole d'Expérimentation Avicole de Tours, Nouzilly, France) and immediately transferred to the PFIE unit (Plateforme d'Infectiologie Expérimentale, INRAE, Nouzilly, France), where they were reared together on floor. At 7 days of age, each chick was orally infected with Salmonella enterica Enteritidis [Strain 775 (LA5 Nal20Sm500), $\left.5 \times 10^{4} \mathrm{cfu} / 0.2 \mathrm{~mL} / \mathrm{chick}\right]$ and the chicks were transferred into four isolators to decrease oro-faecal recontaminations, as described previously
[2], with two isolators for chicks from line $\mathrm{N}$ and two for chicks from line $6_{1}$, each isolator harbouring 30 birds. Sibs and half-sibs were separated between the two isolators for each line, in order to prevent confounding of a putative intra-line genetic effect on Salmonella carriage with the environmental effect of the isolator. Isolators with birds from line $6_{1}$ in Experiment 1 were used for birds from line $\mathrm{N}$ in Experiment 2 in order to prevent confounding of isolator effects with line effects. Then, at $12 \mathrm{dpi}$, animals were euthanized with $\mathrm{CO}_{2}$ according to the French regulation for experimental chickens. Caecal contents were gently collected to ensure that the intestinal mucosa was retained, weighed, transferred into cryotubes, and immediately frozen in liquid nitrogen and stored at $-80{ }^{\circ} \mathrm{C}$ until use. All animal procedures were approved by the Ethic committee (APAFIS\#5833$2016062416362298 \mathrm{v} 3$ ) and authorised by the French Government.

\section{DNA extraction}

Individual caecal DNA was extracted from $\sim 200 \mathrm{mg}$ of frozen caecal contents, as previously described [27]. Samples from Experiments 1 and 2 (see Fig. 1) were processed at the same time. In brief, samples were incubated at $70{ }^{\circ} \mathrm{C}$ for $1 \mathrm{~h}$ with $250 \mu \mathrm{L}$ of guanidine thiocyanate buffer [ $4 \mathrm{M}$ guanidine thiocyanate $-0.1 \mathrm{M}$ Tris $(\mathrm{pH}$ 7.5 ) and $40 \mu \mathrm{L}$ of $10 \% \mathrm{~N}$-lauroyl sarcosine $-0.1 \mathrm{M}$ phosphate buffer ( $\mathrm{pH} 8.0)]$ and $500 \mu \mathrm{L}$ of $5 \% N$-lauroyl sarcosine. One volume $(750 \mu \mathrm{L})$ of $0.1-\mathrm{mm}$-diameter silica beads (Sigma) was added and the tubes were shaken for $10 \mathrm{~min}$ at the maximum speed of a MM200 Mixer Mill (Retsch, Germany). Tubes were vortexed and centrifuged

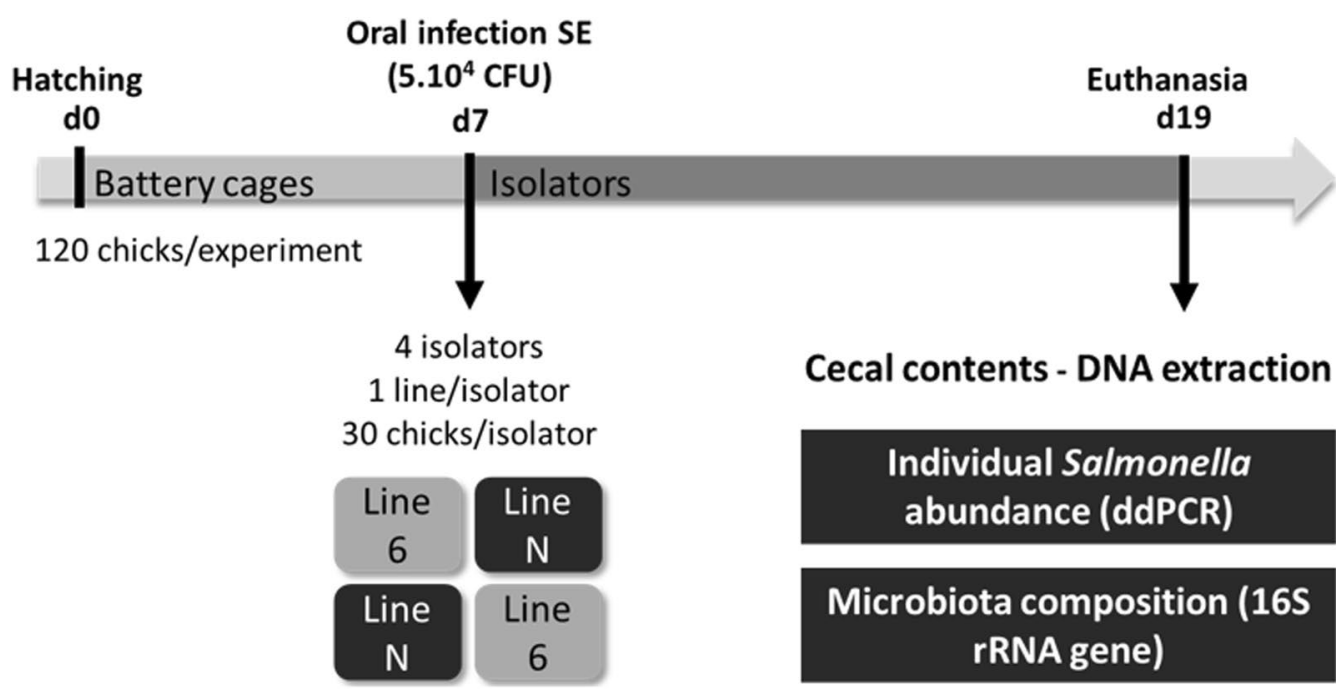

Fig. 1 Experimental design. Two experiments were conducted, one in January 2017 and one in February 2017 for a total of 240 animals from the inbred White Leghorn lines $\mathrm{N}$ and $\sigma_{1}$ 
at $14,000 \mathrm{rpm}$ for $5 \mathrm{~min}$ at $4{ }^{\circ} \mathrm{C}$. After recovery of the supernatant, $30 \mu \mathrm{L}$ of Proteinase $\mathrm{K}$ (Chemagic STAR DNA BTS kit, Perkin Elmer, USA) were added and samples were incubated for $10 \mathrm{~min}$ at $70{ }^{\circ} \mathrm{C}$ in a Multi-Therm shaker at $250 \mathrm{rpm}$ (Benchmark Scientific, USA), then for $5 \mathrm{~min}$ at $95{ }^{\circ} \mathrm{C}$ for enzyme inactivation. The tubes were centrifuged at $14,000 \mathrm{rpm}$ for $5 \mathrm{~min}$ at $4{ }^{\circ} \mathrm{C}$ and the supernatants were transferred into a deepwell plate. The plate was loaded onto the nucleic acid workstation Chemagic STAR (Hamilton, Perkin Elmer, USA) and DNA extraction was performed with the Chemagic STAR DNA BTS kit (Perkin Elmer, USA) by the @BRIDGe platform (INRAE, Jouy-en-Josas, France) according to the manufacturer's instructions. DNA concentration was measured by fluorometric quantification (Qubit) and DNA samples were stored at $-20^{\circ} \mathrm{C}$.

\section{Quantification of $S$. Enteritidis by ddPCR}

Individual abundances of SE in caecal contents at day 19 were obtained by Droplet Digital PCR using the QX200 Droplet Digital PCR system (Bio-Rad) on the @BRIDGe platform (INRAE, Jouy-en-Josas, France). Each DNA sample was diluted 1:2 and 1:5 in a final volume of $5 \mu \mathrm{L}$ to be under PCR saturated conditions. Droplets were generated using samples of $20 \mu \mathrm{L}$ taken from the amplification mix containing genomic DNA (15,000-20,000 droplets per sample). Amplification targeted a region of the InvA gene specific to SE using specific primers (forward: 5'-GCGTTCTGAACCTTTGGT-3', reverse: $5^{\prime}$-CGTTCGGGCAATTCGTTA-3') as described by Daum et al. [28] (see Additional file 1). PCR cycling conditions started with an enzyme activation step at $95{ }^{\circ} \mathrm{C}$ for $10 \mathrm{~min}$, followed by 40 cycles at $94{ }^{\circ} \mathrm{C}$ for $30 \mathrm{~min}$ and $60{ }^{\circ} \mathrm{C}$ for $1 \mathrm{~min}$, and ended by an enzyme deactivation step at $98{ }^{\circ} \mathrm{C}$ for $10 \mathrm{~min}$. For all cycling steps, we used a $2.5^{\circ} \mathrm{C} / \mathrm{s}$ ramp rate.

For each sample, the number of copies of Salmonella per $g$ of caecal content was calculated from the average number of copies of Salmonella per $\mu \mathrm{L}$ of the two dilutions, assuming that each amplified copy of the InvA gene corresponds to one Salmonella bacterium. Data were analysed with a log transformation of the copies of Salmonella. A Levene's test was performed to test for differences in variation between groups. Analyses of variance (Anova) were performed to test the significance of differences in the number of copies of Salmonella between conditions (testing the effects "line", "sex", and "experiment") using the Anova function from the car package (version 3.5-5) in the $\mathrm{R}$ software version 3.5.1 (Type II sum of squares), with a heteroscedasticity-corrected coefficient covariance matrix (white.adjust $=$ TRUE) to account for differences in variances between groups.

\section{PCR and sequencing of the 16S rRNA genes}

Samples from Experiments 1 and 2 were sequenced at the same time. Amplification of the V3-V4 hyper-variable region of the $16 \mathrm{~S}$ rRNA coding gene was performed on the INRAE @BRIDGe platform. Universal V3-V4 primers (see Additional file 1) were used for the first PCR reaction. PCR cycling conditions were as follows: an initial denaturation step $\left(94{ }^{\circ} \mathrm{C}\right.$ for $\left.10 \mathrm{~min}\right), 35$ cycles of amplification $\left(94{ }^{\circ} \mathrm{C}\right.$ for $1 \mathrm{~min}, 68^{\circ} \mathrm{C}$ for $1 \mathrm{~min}$ and $72{ }^{\circ} \mathrm{C}$ for $1 \mathrm{~min}$ ) and a final elongation step at $72{ }^{\circ} \mathrm{C}$ for $10 \mathrm{~min}$. Amplicons were then purified using magnetic beads (Clean NA, GC biotech B.V., The Netherlands). The DNA concentration was controlled using a Nanodrop spectrophotometer (Thermo Scientific, USA). In the second PCR, samples were multiplexed and another pair of primers was used (see Additional file 1) with the following PCR steps: an initial denaturation step $\left(94{ }^{\circ} \mathrm{C}\right.$ for $10 \mathrm{~min}), 12$ cycles of amplification $\left(94{ }^{\circ} \mathrm{C}\right.$ for $1 \mathrm{~min}, 65^{\circ} \mathrm{C}$ for $1 \mathrm{~min}$ and $72^{\circ} \mathrm{C}$ for $1 \mathrm{~min}$ ) and a final elongation step at $72{ }^{\circ} \mathrm{C}$ for $110 \mathrm{~min}$. Amplicons were purified and the DNA concentration was controlled as described for the first PCR reaction. One run on an Illumina MiSeq was used to sequence amplicons $(2 \times 250$ paired-end reads $)$ according to the standard protocol.

\section{Bioinformatic and statistical analyses}

Identification of OTU was performed using the FROGS pipeline [29]. The FastQC program was used to control quality and the Cutadapt program to find and remove adapter sequences from sequencing reads. R1 and R2 reads were merged and filtered (at Phred $\geq$ Q20) with the Flash program [30]. OTU were identified with the Swarm program [31]. Chimera OTU were removed by the VSEARCH program [32], and taxa filtering was performed with a minimum relative abundance threshold of $0.005 \%$ as proposed by [33]. Finally, phylogenetic affiliations were identified based on the Silva database by using the blastn + program [34]. OTU representing less than $0.5 \%$ of the global reads and samples with less than 10,000 reads were removed. For all the analyses involving diversity measures, rarefaction was performed using the rarefy_even_depth function in the phyloseq (1.24.2) package.

The phyloseq (1.24.2) and vegan (2.5-3) packages in $\mathrm{R}$ were used to perform diversity analyses on the rarefied data. Alpha diversity and beta diversity were measured based on the Shannon index and the Whittaker index, respectively. Anova were performed to test the significance of differences in alpha and beta diversities between conditions (line, sex, experiment, and isolator). Bray-Curtis dissimilarity was evaluated using the env_fit method by plotting in a non-metric multidimensional 
scaling representation. Permutational multivariate analyses of variance (Permanova) were used to test for significance of differences in diversity between lines, experiment, isolator, and sex.

The $\mathrm{R}$ package metagenomeSeq (1.24.1) was used on the unrarefied data to identify differentially abundant (DA) OTU between lines $\mathrm{N}$ and $6_{1}$ and between high and low carriers from line $6_{1}$. After normalisation of the OTU table, the model was fitted using the fitZIG method by including experiment, isolator, and sex as cofactors. Heatmaps were built on significant DA OTU using the function plotMRheatmap. Then, OTU were aggregated by family and genus with the command aggregateByTaxonomy to identify DA families and genera. Functional gene families and the MetaCyc pathway were predicted using the PICRUST2 package. DA KEGG orthologs (KO) and DA MetaCyc pathways were identified using the $\mathrm{R}$ package DESeq2 (1.26.0). DA MetaCyc pathways were aggregated at the super-pathway level based on the MetaCyc database [35].

\section{Results}

\section{Abundance of $S$. Enteritidis in caecal contents}

After oral Salmonella infection at seven days of age, the chickens showed no clinical signs as expected with this dose of SE. The SE abundances in caecal content at $12 \mathrm{dpi}$ from 228 chickens were well characterized by ddPCR, with an average of 3.38 and $2.55 \log 10$ copies of DNA target sequences per $g$ of caecal content for line $6_{1}$ and line $\mathrm{N}$, respectively (Fig. 2). Since the Levene's test showed differences in variance between groups $(P<0.001)$, we performed Anova using heteroscedasticity-corrected type II sum of squares by including line, experiment, and sex as factors, which revealed a significant difference $(\mathrm{P}<0.001)$ in individual Salmonella carriage (ISC) between the two lines (Table 1) and (see Additional file 5: Table S1), which was also found when considering each experiment separately (Table 1 ). In addition, the ISC was found to be more variable in line $6_{1}$ than in line $\mathrm{N}(\mathrm{P}<0.001)$, with standard deviations of 1.3 and 0.7 , respectively. The higher ISC variation in line $6_{1}$ was more marked in Experiment 1 than in Experiment $2(\mathrm{P}<0.001)$, with standard deviations of 1.61 vs. 0.84 (Fig. 2). The average ISC did not differ significantly between males and females (see Additional file 5: Table S1). The average ISC was significantly different between the two experiments $(\mathrm{P}<0.001)$ for line $\mathrm{N}(2.2 \pm 0.7$ in Experiment 1 vs. $2.9 \pm 0.4$ in Experiment 2) but not for line $6_{1}$. Differences between isolators were not significant for line $6_{1}$ in Experiment 1, but there were significant differences between isolators for line $6_{1}$ in Experiment 2 and between isolators for line $\mathrm{N}$ in Experiments 1 and $2(\mathrm{P}<0.01)$.

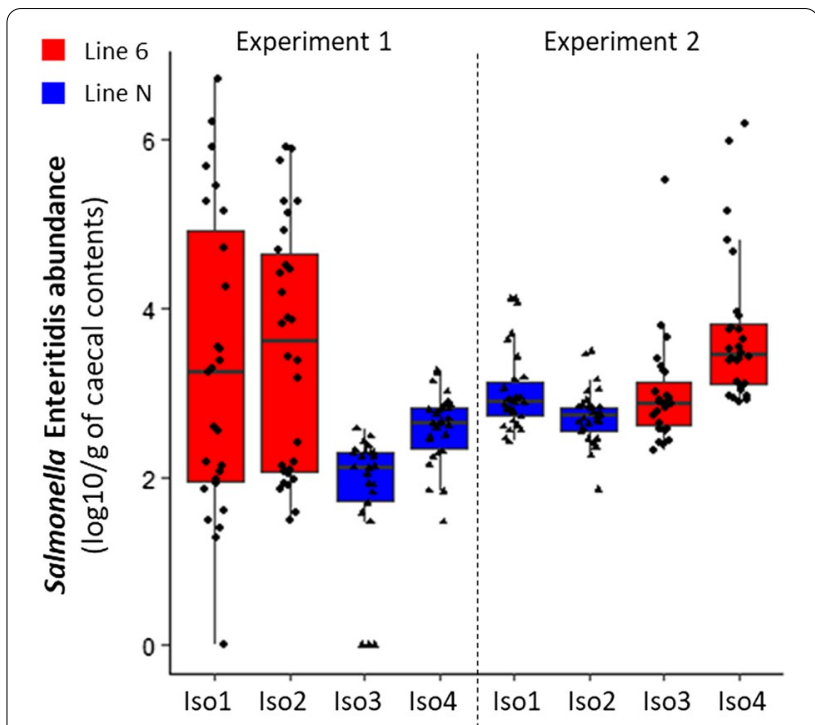

Fig. 2 Salmonella Enteritidis abundance in caecal contents at 12 days post infection. Salmonella Enteritidis abundance at 12 days post infection in caecal contents of chickens from lines $\mathrm{N}$ and $\sigma_{1}$ infected with S. Enteritidis (log 10/g of caecal contents) by experiment and isolator. Salmonella carriage is significantly different between lines and between experiments. In line $\sigma_{1}$, the carriage is more variable between chickens, in particular for Experiment 1

In Experiment 1 for line $6_{1}$, hierarchical clustering identified two extreme groups of 15 chickens referred to as low and high carriers and for which usable information on microbiota was available. The mean abundance was $1.8 \pm 0.6$ and $5.2 \pm 0.6 \log 10$ copies of DNA per $g$ of caecal content for the low and high carrier groups, respectively.

\section{Structure and diversity of the bacterial communities}

Metadata, unrarefied OTU and the corresponding taxonomic classifications are in Additional files 2, 3 and 4, respectively. Due to strict controls on DNA quality after extraction and amplification, samples with at least $10 \mathrm{ng}$ of DNA from 228 of the 240 chickens were sequenced. Sequencing resulted in $8,300,144$ reads, with on average 32,464 reads per sample. From these reads, 617 OTU were identified, with percentages of affiliation of 99 and $65 \%$ at the family and genus levels, respectively. Samples with less than 10,000 total reads were removed, leaving data on 182 chickens, 86 and 95 from lines $6_{1}$ and $\mathrm{N}$, respectively. The excluded samples were distributed across lines, genders, experiments, and isolators.

At the phylum level, Firmicutes dominated the bacterial composition, followed by Proteobacteria and Bacteroidetes (Fig. 3a). At the family level, we observed a predominance of Lachnospiraceae and Ruminococcaceae, 
Table 1 Mean and standard deviation of Salmonella Enteritidis abundance in caecal contents by line and experiment 12 days post infection and significance (P-value) of line effects based on Anova type II sum of squares test using all factors

\begin{tabular}{|c|c|c|c|c|c|c|}
\hline \multirow[t]{2}{*}{ Line } & \multicolumn{2}{|c|}{ Experiment 1} & \multicolumn{2}{|c|}{ Experiment 2} & \multicolumn{2}{|c|}{ Both experiments } \\
\hline & Number & Mean \pm SD & Number & Mean \pm SD & Number & Mean \pm SD \\
\hline N & 56 & $2.21 \pm 0.74$ & 60 & $2.86 \pm 0.43$ & 116 & $2.55 \pm 0.68$ \\
\hline 6 & 57 & $3.41 \pm 1.61$ & 55 & $3.35 \pm 0.84$ & 112 & $3.38 \pm 1.29$ \\
\hline P-value & & $2.03 e-06$ & & $2.12 e-04$ & & $3.45 e-09$ \\
\hline
\end{tabular}

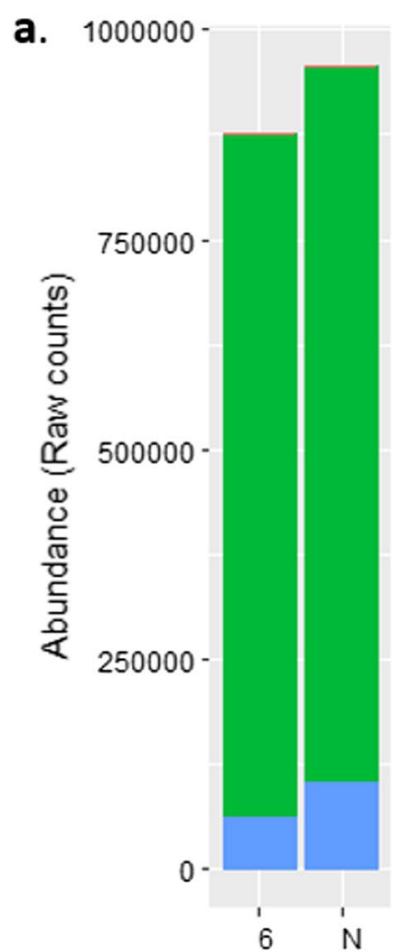

Phylum

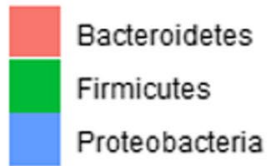

b.

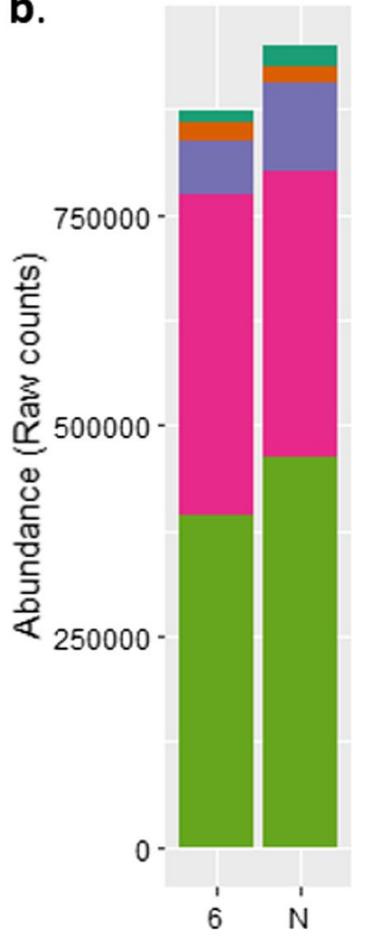

Family

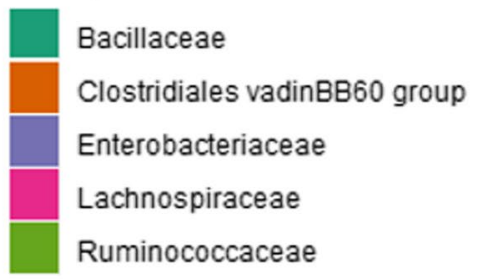

C.

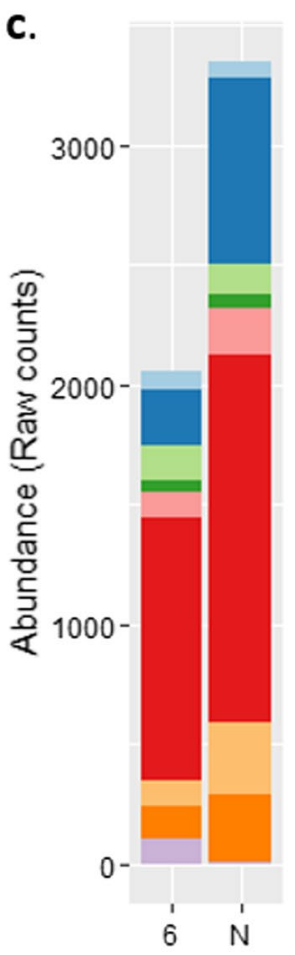

\begin{tabular}{l} 
Family \\
\hline Barnesiellaceae \\
Christensenellaceae \\
Clostridiaceae 1 \\
\hline Enterococcaceae \\
Erysipelotrichaceae \\
Lactobacillaceae \\
Peptostreptococcaceae \\
Pseudomonadaceae \\
Streptococcaceae
\end{tabular}

Fig. 3 Microbiota composition at phylum and family levels for lines $\mathrm{N}$ and $\sigma_{1}$. Microbiota composition for lines $\mathrm{N}$ and $\sigma_{1}$ using raw counts after rarefaction at the phylum level (a), at the family level for the most abundant taxa (b) and at the family level for the least abundant taxa (c). The most abundant phylum is Firmicutes followed by Proteobacteria and Bacteroidetes. There is no significant difference at the phylum level between lines. At the family level we observed, with decreasing abundances, Ruminococcaceae and Lactobacillaceae, followed by Enterobacteriaceae, Clostridiales and Bacillaceae 
followed by Enterobacteriaceae, Clostridiales and Bacillaceae (Fig. 3b) and (see Additional file 5: Table S2).

Analysis of the alpha diversity with the Shannon index did not show significant differences between lines, experiments, or sexes (Fig. 4) and (see Additional file 5: Table S3). Using the Whittaker index, we observed a significant difference in beta diversity between experiments $(\mathrm{P}<0.0001)$, with 0.24 for Experiment 1 vs. 0.20 for Experiment 2. However, we did not observe a difference in beta diversity between lines ( 0.20 for both lines). Highand low-carriers from line 61 did not significantly differ in alpha diversity and richness but did in beta diversity (Fig. 4) and (see Additional file 5: Table S3).

\section{Non-metric multidimensional scaling and differentially abundant OTU between lines}

Analysis of the beta diversity using Bray-Curtis dissimilarity and an NMDS representation (Fig. 5) showed that the microbiota composition clearly clustered by line. This clustering was observed for both experiments but in particular for Experiment 1 (Fig. 5). The Permanova analysis confirmed this line effect $(\mathrm{P}<0.001)$ after correcting for experiment, isolator, and sex effects, regardless of whether the data from Experiments 1 and 2 were merged or analysed separately (see Additional file 5: Table S4). The microbiota composition also differed significantly between the two experiments and between isolators but not between males and females (Additional file 5: Table S4).

Among the 617 identified OTU, 390 were significantly differentially abundant (DA) between lines across the two experiments, after adjusting for isolator effects (see Additional file 5: Table S5). When the two experiments were analysed separately, the heatmap showed a more strongly defined clustering for Experiment 1, with 388 DA OTU between lines (Fig. 5), than for Experiment 2, with 284 DA OTU. In total, 187 common DA OTU were found between the two experiments.

The 617 OTU were aggregated in order to compare compositions at higher taxonomic ranks, which led to 14 families and 51 genera. Nine DA families and 31 DA genera were identified between the two lines over the two experiments, after adjusting for isolator effects (Fig. 6) and (see Additional file 5: Table S6). For Experiments 1 and 2, nine and five DA families and 24 and 29 DA genera were identified, respectively (Fig. 6). At these levels of aggregation, families and genera that were significantly DA between the two lines were not the same for the two experiments, and some were inversely abundant. Nevertheless, in both experiments, the Christensenellaceae family was more abundant in the resistant line N. At the genus level, Tyzzerella 3, Lachnoclostridium, Marvinbryantia, Ruminococcaceae UCG-013 were more abundant in line $6_{1}$ (susceptible) and Ruminococaceae UGC-004, Ruminococcus 1, Pseudomonas, Pseudoflavonifractor, Christensenellaceae R-7 group and Ruminococcaceae UGC-014 were more abundant in line $\mathrm{N}$ (resistant).

\section{High and low carriers from line $6_{1}$ and the family Christensenellaceae}

An NMDS representation for Experiment 1 was used to assess a potential difference in microbiota composition 


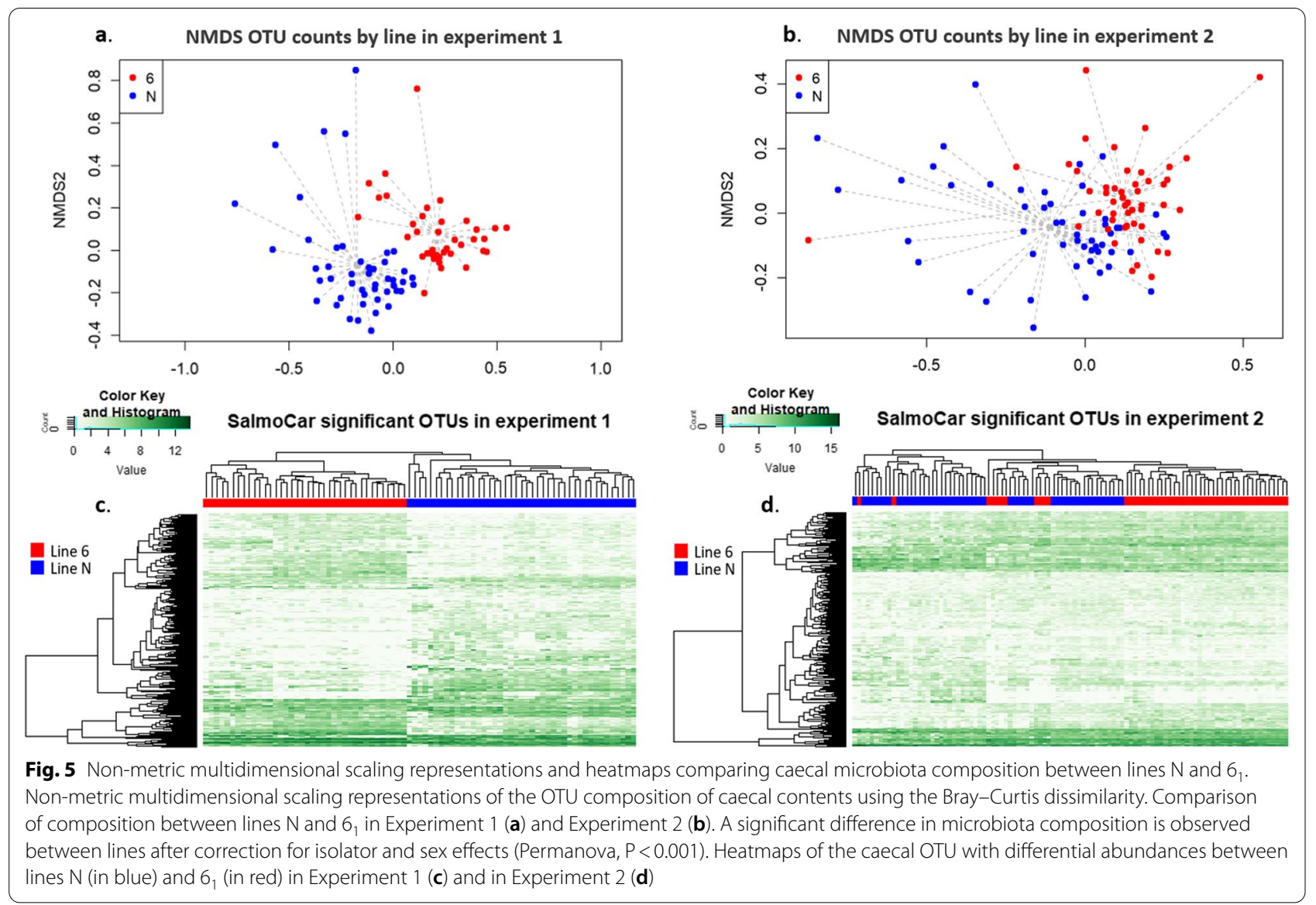

between high and low carriers from line $6_{1}$ (Fig. 7a). Experiment 1 was used for this analysis because it had the largest variability in individual Salmonella carriage, which allowed for the identification of high and low carriers. In Experiment 1, the Permanova analysis showed a significant $(\mathrm{P}<0.01)$ difference in microbiota composition between the high and low carriers. DA analysis using the metagenomeSeq package identified 39 DA OTU between the high and low carriers (Fig. 7b) and (see Additional file 5: Table S7). After aggregation, one DA family (Christensenellaceae) and three DA genera (Ruminococcaceae NK4A214, Ruminiclostridium 5, and Christensenellaceae R-7 groups) were identified (Fig. 7c) and (see Additional file 5: Table S8).

A comparison of results from the DA analyses between lines, on the one hand, and between high and low carriers, on the other hand, led to the identification of one common DA family, i.e. Christensenellaceae, which was more abundant in low carriers than in high carriers from line $6_{1}$ and was also more abundant in chickens from line $\mathrm{N}$, which are more resistant (i.e. low carrier) to Salmonella. The same was observed for the DA genus Christensenellaceae $R-7$ group, which was more abundant in low carriers and in the resistant line $\mathrm{N}$ in both experiments. Regression analysis identified a significant correlation $\left(\mathrm{P}\right.$-value $\left.=4.11 \mathrm{e}^{-06}\right)$ between ISC and Christensenellaceae abundance.

\section{Functional gene prediction}

By using the PICRUST2 software, 4954 KEGG orthologs (KO) and 323 pathways were predicted based on the OTU table. The DESeq2 package identified 1590 DA KO and 69 DA pathways between lines $\mathrm{N}$ and $6_{1}$, after adjustment for the effect of experiment, (false discovery rate $($ FDR $)<0.01)$. For line $6_{1}$, the analysis between high and low carriers in Experiment 1, after adjustment for isolator effects, resulted in $507 \mathrm{DA} \mathrm{KO}$ and $75 \mathrm{DA}$ pathways (FDR $>0.01$ ). The 64 common DA pathways between lines $\mathrm{N}$ and $6_{1}$ and between high and low carriers from line $6_{1}$ were aggregated at the super-pathway level (Fig. 8) and (see Additional file 5: Table S9).

\section{Discussion}

Using a model of Salmonella infection on chickens placed in isolators, which limits animal to animal recontaminations and animal reinfection and, thus, homogenisation 
Line $6_{1}$

Line $\mathrm{N}$

Family level

Genus level
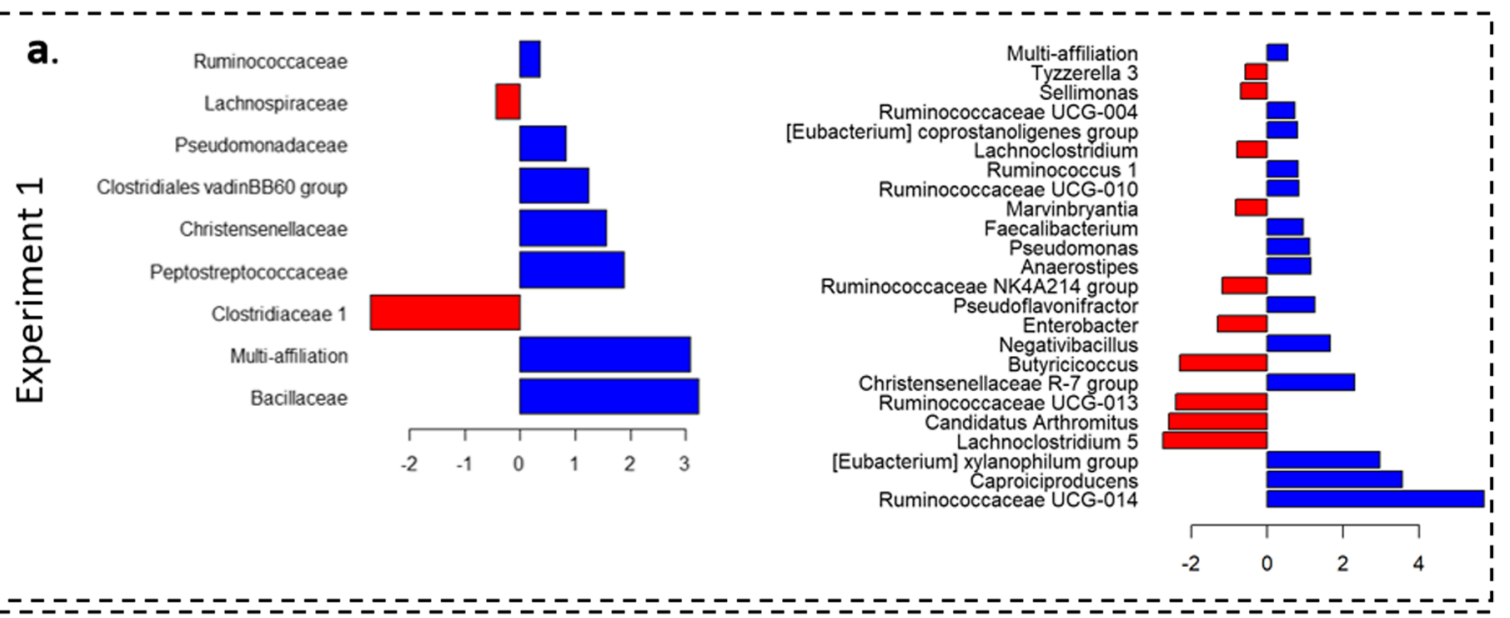

b.

b.

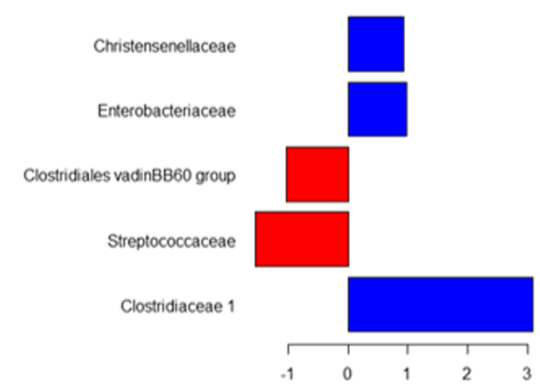

[Ruminococcus] gauvreauli group

[Eubacterium] coprostanoligenes group

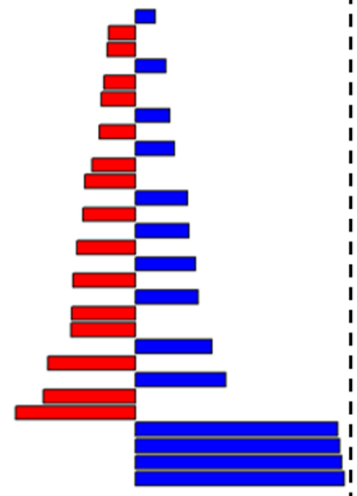

[Eubacterium] Blaut

s.
scherichia-shige

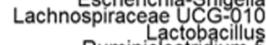

Ruminiclostridlum
Christensenellaceae R-7 grou

Ruminococcaceae UCG-00

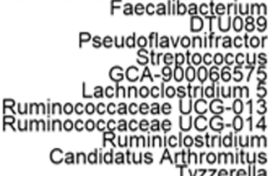

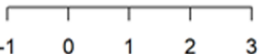

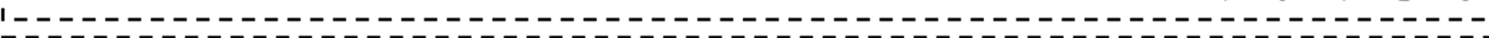

C.
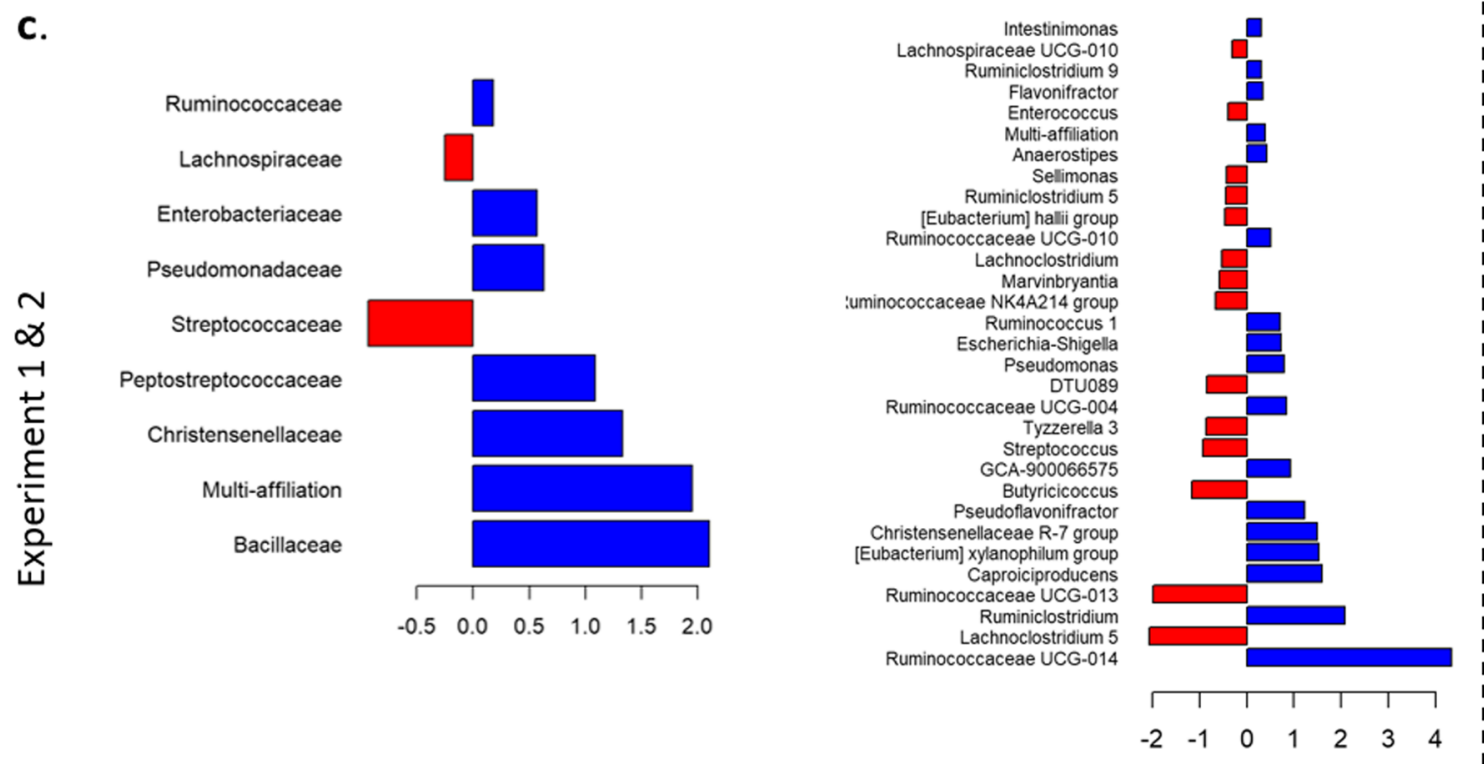

Fig. 6 Differentially abundant families and genera between lines $N$ and $\sigma_{1}$ in Experiments 1 and 2. Differentially abundant families and genera between lines $N$ and $\sigma_{1}$ for Experiment 1 (a), Experiment 2 (b), and Experiments 1 and 2 together (c). Representation of the log fold-changes for the comparison between lines (blue indicates greater abundance in line $\mathrm{N}$, red greater abundance in line $6_{1}$ ) 
a.

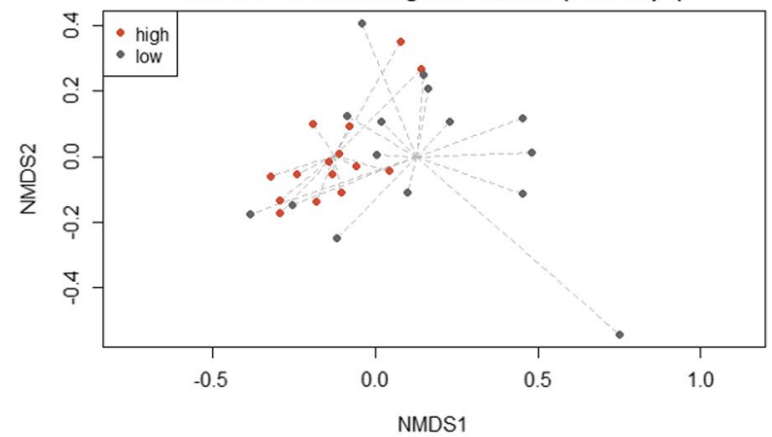

C.

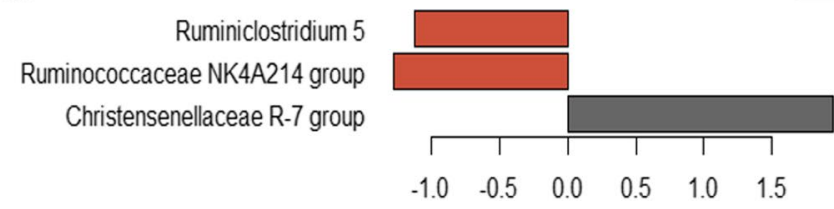

b.

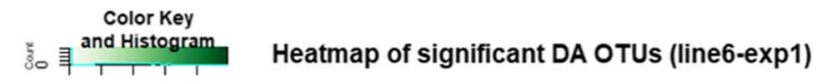

$\begin{array}{lllll}7 & 1 & 1 & 1 & 1 \\ 0 & 2 & 4 & 6 & 8\end{array}$

Value
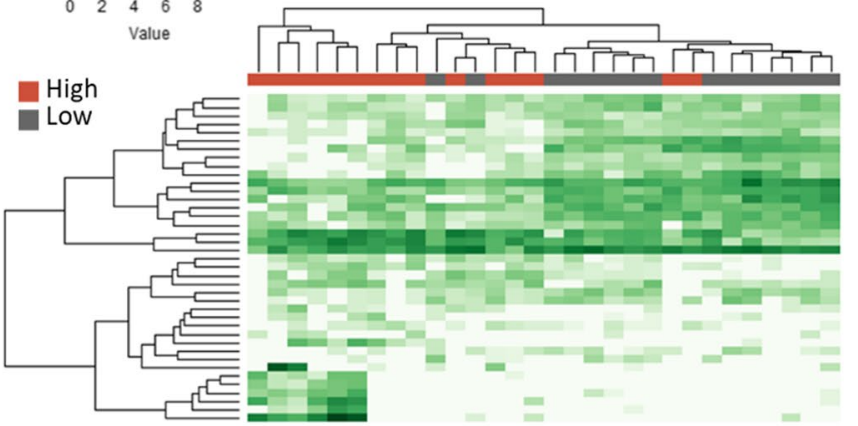

Fig. 7 Non-metric multidimensional scaling representation, heatmap, and significant differentially abundant genera for high and low carriers (line $\sigma_{1}$ - exp1). a Non-metric multidimensional scaling representation of the caecal microbiota of high and low carriers from line $\sigma_{1}$ (Bray-Curtis dissimilarity). High and low carriers are significantly different (Permanova, P-value $<0.01$ ); caecal microbiota from different isolators are not significantly different (Permanova, P-value > 0.5). b Heatmap representation of the 39 differentially abundant OTU between high-and low-carriers

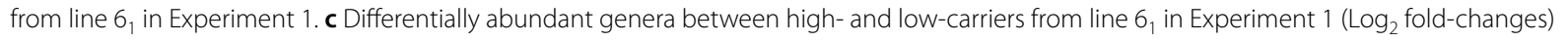

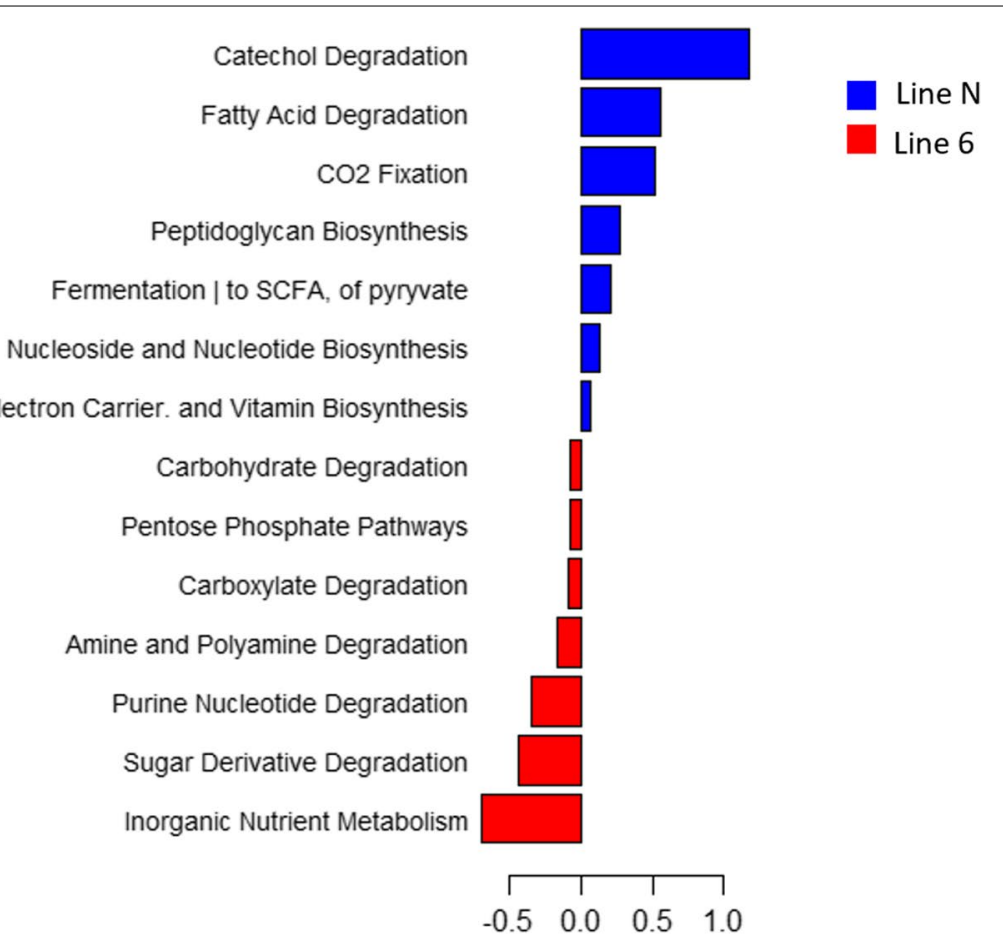

Fig. 8 Common differentially abundant super-pathways between lines $N$ and $\sigma_{1}$ and between high and low-carriers from line $\sigma_{1}$. Common differentially abundant super-pathway (MetaCyc database-DESeq2) in the comparison of caecal microbiota between lines $\mathrm{N}$ and $\sigma_{1}$ and between high and low carriers from line $\sigma_{1}$. Representation of the $\log _{2}$ fold-changes for the comparison between lines (blue indicates greater abundance in line $\mathrm{N}$ and red greater abundance in line $6_{1}$ ) 
of individual Salmonella carriage [2], we confirmed that individual Salmonella carriage varied both with the host genetics and microbiota composition. We identified differences in gut microbiota that were associated both with genetic line (N vs. $6_{1}$ ) and the individual Salmonella carriage status (high vs. low ISC carriers). Finally, the caecal microbiota taxonomic analysis identified taxa and metabolism pathways that may be associated with Salmonella carriage.

In both Experiments 1 and 2, line $\mathrm{N}$ was more resistant than line $6_{1}$ (based on the ISC phenotype), and the microbiota taxonomic composition also differed significantly between the two lines. Nevertheless, ISC was higher in Experiment 2, especially for line $\mathrm{N}$, and the OTU that were DA between lines were not entirely the same between Experiments 1 and 2. Differences in microbiota composition between the two experiments might be due to the differences in environment. Although conditions were strictly controlled to be identical between the two experiments, slight differences in the environment may occur, resulting in different microbial compositions in the environment, which affects the primo-colonisation of the gut by bacteria in new-born chicks. Two studies have reported differences in the primo-colonisation of the intestinal tract between chickens, even when they are reared together in the same environment [36, 37]. Differences in microbiota composition before infection could affect microbiota composition after infection. Because of differences in ISC and microbiota composition between the two experiments, we will focus only on the results that were validated in both experiments in the following section.

\section{Impact of the chicken line on individual Salmonella Enteritidis carriage and microbiota composition}

Using different infection models, previous studies have shown the impact of host genetics on Salmonella carriage and significant differences in SE carriage between commercial or local chicken breeds [38-40]. Several candidate genes have been shown to be associated with resistance to SE carriage, such as SLC11A1 and TLR4 [41-43]. Our results with lines $N$ and $6_{1}$ confirm previously reported results for the same two lines without using isolators and with on-floor grouped rearing [3, 44], which confirm that line $\mathrm{N}$ is a lower SE carrier than line $6_{1}$. In addition, several QTL have been identified in different populations that derive from $\mathrm{N} \times 6_{1}$ crosses [3-5]. Interestingly, our two independent experiments confirmed a larger variability in ISC for line $6_{1}$ than for line $\mathrm{N}$, which raises new hypotheses, i.e. whether this difference in variability in ISC is caused by genetic differences between the lines, or by a differences in microbiota composition.
In the two experiments conducted here, the significant difference in caecal microbiota composition between lines $N$ and $6_{1}$ at 12 dpi suggests the presence of host genetic control of this composition. For each experiment, birds from the two lines were hatched in the same environment and at the same time, and raised together until the experimental infection, such that the initial microbial environment was similar for all birds tested before infection. Thus, differences in caecal microbiota composition between lines cannot be attributed to differential exposures to environmental microbes before infection. Furthermore, for Experiment 1, we observed no significant difference in microbiota composition between the two isolators that were used for a given line $[\mathrm{P}>0.1$ (see Additional file 5: Table S4)]. Thus, in Experiment 1 we cannot associate differences in microbiota composition between the two lines with an effect of the isolator environment. In contrast, in Experiment 2, a significant difference in microbiota composition was observed between the two isolators used for a given line $[\mathrm{P}<0.01$ (see Additional file 5: Table S4)]. One can argue that the isolators were populated by different microbial populations, which could in turn influence the caecal microbiota composition after infection, but in this study the isolators were sterilized before each experiment. In addition, although the isolators used for each line were switched between the two experiments, observed differences in caecal microbiota composition between the two lines were similar for the two experiments. Moreover, since the isolators decrease oro-faecal recontamination of commensal microbiota and of pathogenic bacteria, the caecal microbiota composition of each chicken matured in isolation from the impact of the other birds once the animal has been put into the isolator.

Since we analysed caecal microbiota composition after infection, we cannot exclude that it was affected by the infection with Salmonella. Does the higher susceptibility of line $6_{1}$, with a larger number of Salmonella in the caeca, cause a shift in microbiota composition? Several studies have shown that SE infection has a very low impact on the composition of the caecal microbiota [23, 45-47], even if some differences in taxa abundance have been identified between infected and uninfected chicken $[47,48]$. Thus, we hypothesize that, in our study also, SE infection had a weak impact on microbiota composition.

In a study of Campylobacter carriage between lines $\mathrm{N}$ and $6_{1}$, microbiota were transferred from the resistant to the susceptible line [49]. Although microbiota composition did not differ between the donor birds from the $\mathrm{N}$ and $6_{1}$ lines at 21 days of age, the genetic line of the recipient had a significant impact on microbiota composition in the recipient after transplantation, while the donor line of the transplanted microbiota did not. This supports 
our hypothesis that the host genetics has a strong impact on the composition of the microbiota in lines $\mathrm{N}$ and $6_{1}$ at 3 weeks after hatching and that genetic differences between lines N and $6_{1}$ might indirectly influence Salmonella carriage by impacting the microbiota composition. However, this does not exclude other potential pathways, in particular those involving host immunity.

In chickens, as well as in other livestock species and in humans, host genetic control of intestinal microbiota composition is well documented. In studies on humans, the abundance of some bacteria that are known to be associated with host immunity is heritable and host candidate genes have been identified [50]. In chickens, some studies have identified differences in microbiota composition between genetic lines, for instance between two lines that differ in their susceptibility to bacterial infections [51], between two divergent lines for body weight [52], and between four commercial lines and an indigenous Indian breed [53]. At least two studies have reported moderate estimates of heritability for bacteria family abundances and have detected several QTL involved in the control of these bacterial abundances by the host $[24,54]$.

\section{Relationships of caecal microbiota with individual Salmonella Enteritidis carriage}

Colonisation and adhesion of commensal bacteria on the mucosal epithelium constitute a protective biofilm due to their competitive exclusion (CE) function. Studies affirm that CE is currently the best approach to decrease Salmonella colonization in commercial production of chickens [10]. In our study, all animals were experimentally infected and signature bacteria were identified by comparing chickens with differences in individual Salmonella carriage between or within lines. Our hypothesis is that the abundance of commensal, potentially competitive bacteria, is higher in the microbiota of resistant chickens, thus preventing colonisation of Salmonella in the intestinal tract. Host genetics could, in part, control this abundance of competitive bacteria.

From our results, it appears that the difference in ISC between lines and the larger variability of ISC in line $6_{1}$ cannot be related to a difference in beta- or alphadiversity of the caecal microbiota between the two lines. Other studies that compared chickens from different breeds or lines found no significant differences in the Shannon index between birds that were infected with SE versus those that were not at $14 \mathrm{dpi}[47,55]$ or at $10 \mathrm{dpi}$ [23]. These observations corroborate our findings and lead us to the conclusion that neither infection with SE nor the level of SE carriage affect OTU diversity in the caecal microbiota. However, we identified a significant difference in beta diversity between high and low carriers from line $6_{1}$, in that microbiota of high carriers were more similar to each other than those of low carriers. This raises the question whether the higher level of Salmonella drives the microbiota to a more similar composition. Or are high carriers more susceptible to Salmonella because some shared characteristics of their microbiota lead to a less efficient competitive exclusion? These questions remain open, as inbred lines $\mathrm{N}$ and $6_{1}$ are not fully homozygous, so that residual genetic variations within line $6_{1}$ may explain these differences in caecal microbiota beta diversity.

Even in the absence of differences in alpha- or betadiversity, the DA OTU that were identified between the two lines could be associated with differences in ISC (Fig. 6). Likewise, the DA OTU that were identified between high and low carriers from line $6_{1}$ could be associated with ISC (Fig. 7).

We observed that the Christensenellaceae family was more abundant in low Salmonella carriers (Figs. 6 and 7). This bacteria family is associated with a beneficial impact on health in humans and in mice [56-59]. Interestingly, it has been shown that Christensenellaceae is one of the most heritable bacterial families of the human intestinal microbiota [56]. This leads us to formulate the hypothesis that host genetic differences between lines $\mathrm{N}$ and $6_{1}$ cause differences in Christensenellaceae abundance, which in turn could affect Salmonella resistance. However, to our knowledge, the heritability of the abundance of Christensenellaceae has not been assessed in chickens. Similarly, the family Enterobacteriaceae was more abundant in the resistant line (Fig. 6). These bacteria are in competition with Salmonella for oxygen [60] and for the use of nutrients such as iron [61] and are also able to produce bacteriocin that inhibits the proliferation of $\mathrm{Sal}$ monella [60]. Oxygen respiration by competitive bacteria such as Enterobacteriaceae, leads to anaerobisation of the lumen and, thus, a decrease in the capacity of Salmonella colonisation [60]. Our results are compatible with this hypothesis. Finally, the Ruminococcaceae family was less abundant in the susceptible line $6_{1}$ (Fig. 6). It has been shown that a decrease in Ruminococcaceae can be associated with an increase in inflammation [62] and thus an increase in Salmonella competition [63].

Based on the imputed putative functions associated with the DA OTU, based on known databases, some of these DA OTU harboured striking functions. Interestingly, several of the potential functions associated with the DA OTU may explain the observed differences in ISC between lines and between high and low carriers within line $6_{1}$. Although very speculative since it is based on an indirect, in silico approach, the significant functions identified are highly consistent with known mechanisms of resistance to Salmonella. First, the short-chain fatty 
acids (SCFA) metabolic pathway may be more abundant in both the resistant line $\mathrm{N}$ and in low carriers from the susceptible line $6_{1}$. Consequently, the production of SCFA could be associated with low Salmonella carriage. Many studies have shown that the production of SCFA by the intestinal microbiota, such as butyrate, has beneficial effects for the host [64-66]. In chickens, butyric acid can decrease inflammation [67], which is unfavourable for Salmonella [63]. More specifically, it has been shown that increasing SCFA in in vitro cultures of avian intestinal cells decreases the pathogenicity of Salmonella by blocking its entry into the host [68]. Thus, line $\mathrm{N}$ and the low carriers from line $6_{1}$ could carry a beneficial microbiota for Salmonella resistance. Second, the catechol degradation pathway may be more abundant in both the resistant line $\mathrm{N}$ and in the low Salmonella carriers from the susceptible line $6_{1}$. Salmonella have the ability to produce auto-inducers 3 (AI-3), which have a similar chemical structure as the catecholamine from the catechol family $[69,70]$. In chickens and pigs, treatment with a catecholamine such as norepinephrine, is known to increase Salmonella enterica colonization in the host and Salmonella spread in the environment [71, 72]. Catechol also plays a role in quorum sensing signalling for the production of biofilm, thus increasing the virulence of Salmonella during host infection $[69,73]$ and the capacity of Salmonella to persist on eggs or meat [74]. Thus, the capacity of microbiota for catechol degradation in line $\mathrm{N}$ and in the low carriers from line $6_{1}$ could be beneficial for Salmonella resistance.

Nevertheless, bacteria that are potentially beneficial to health have also been found with a higher abundance in the susceptible line. For example, the Blautia genus, which is more abundant in line $6_{1}$ in Experiment 2 (not confirmed in Experiment 1), is a butyrate producer [75] and has beneficial anti-inflammatory effects [76]. Thus, looking at individual taxa might not be sufficient, and we suggest that the total balance of beneficial bacteria has an impact on the Salmonella resistance, which supports the relevance of studying the aggregated contributions of several taxa to the same metabolic pathway.

\section{Conclusions}

Our findings show that ISC in caeca and caecal microbiota taxonomic composition differ between the $\mathrm{N}$ and $6_{1}$ inbred lines of chickens. The results also show associations of ISC status (high vs. low carriers) with the abundance of bacterial taxa and metabolic pathways that were previously associated with resistance to SE. Most notably, we identified an overrepresentation of the short chain fatty acids metabolic pathway and the catechol degradation pathway in low carriers, as well as of the Christensenellaceae, Enterobacteriaceae and
Ruminococcaceae families. Based on these observations, we hypothesize that genetic differences between lines $\mathrm{N}$ and $6_{1}$ may influence the level of Salmonella carriage by influencing the abundance of beneficial bacteria. Combining information on host genetics and gut microbiota composition is useful to increase the accuracy of prediction of complex traits such as resistance to pathogens. Our study showed that both caecal microbiota and host genetic background play a role in mechanisms that lead to Salmonella colonization resistance in chickens. Future studies should decipher the host genes that control differences in bacterial abundances in these model lines and study their effects in other genetic backgrounds and environments.

\section{Supplementary Information}

The online version contains supplementary material available at https://doi. org/10.1186/s12711-022-00699-6.

Additional file 1. Feed composition used, primers sequences used for the amplification of the region specific to Salmonella Enteritidis in the invA gene and adapter/primer used in Illumina amplicon library preparation.

Additional file 2. Metadata associated with all samples.

Additional file 3. Unrarefied operational taxonomic unit table. Additional file 4. Taxonomic assignments for operational taxonomic units.

Additional file 5: Table S1. Results of the Anova test on individual Salmonella carriage for line, sex and experiment. Table S2. Proportion of phylum and family types over the 228 caecal microbiota analysed. Table S3. Results of the Anova test on alpha diversity and beta diversity for line, sex, experiment and high- and low-carriers. Table S4. Results of the Permanova on Bray-Curtis distance for experiment, isolators, sex and line. Table S5. Differentially abundant operational taxonomic units (DA OTU) between the $\mathrm{N}$ and $\sigma_{\text {l }}$ lines over the two experiments, when adjusting for isolator effect. Table S6. Differentially abundant genera and families between the $\mathrm{N}$ and 6 , lines over the two experiments, when adjusting for isolator effect. Table S7. Differentially abundant operational taxonomic units (DA OTU) between the high and low carriers (line 6,-Experiment 1), when adjusting for isolator effect. Table S8. Differentially abundant genera and families between high and low carriers (line 6,-Experiment 1) when adjusting for isolator effect. Table S9. Differentially abundant pathways in common for the comparisons between lines $\mathrm{N}$ and $\sigma_{1}$ and between high and low carriers from line 6 , in Experiment 1.

\section{Acknowledgements}

We thank our colleagues from the experimental unit PEAT who raised the parents of the animals studied and provided the chicks used in our study. We also thank colleagues from the experimental unit PFIE, who efficiently monitored the experiments and collected the samples, and colleagues from the SPVB research team, who helped collect the samples.

\section{Authors' contributions}

$A C$ analyzed the data and wrote the manuscript. FC, BB and PV conceived the study. NB, JLC, MNR, DJ and CB performed laboratory assays. PM, NB, FC, JLC performed animal experiments and collected samples. JE and AR supervised bioinformatics and biostatistics analyses. FC, JE, AR, BB and PV contributed and revised the manuscript. All authors read and approved the final manuscript.

\section{Funding}

Joint program of the Institutes Carnot Santé Animale (ICSA) and Pasteur Maladies Infectieuses (PMI). The PhD of Anais Cazals is funded by Région lle de France (50\%) and the Animal Genetics Division of INRAE (50\%). 


\section{Availability of data and materials}

The sequencing data analysed during the current study are available in the NCBI Sequence Read Archive (SRA) database under the Bioproject accession number PRJNA649900. All data generated and analysed during this study are included in this published article and its additional files.

\section{Declarations}

\section{Ethics approval and consent to participate}

All animal procedures have been approved by the Ethics committee (APAFIS\#5833-2016062416362298v3) and authorised by the French Government.

\section{Consent for publication}

Not applicable.

\section{Competing interests}

The authors declare that they have no competing interests.

\section{Author details}

${ }^{1}$ Université Paris-Saclay, INRAE, AgroParisTech, GABI, 78350 Jouy-en-Josas, France. ${ }^{2}$ Mouse Genetics Laboratory, Department of Genomes and Genetics, Institut Pasteur, Paris, France. ${ }^{3}$ Université François Rabelais de Tours, INRAE, UMR ISP, 37380 Nouzilly, France.

Received: 15 April 2021 Accepted: 17 January 2022

Published online: 29 January 2022

\section{References}

1. European Food Safety Authority European Centre for Disease Prevention and Control. The European union one health 2019 zoonoses report. EFSA J. 2021;19:e06406.

2. Menanteau P, Kempf F, Trotereau J, Virlogeux-Payant I, Gitton E, Dalifard J, et al. Role of systemic infection, cross contaminations and super-shedders in Salmonella carrier state in chicken. Environ Microbiol. 2018;20:3246-60.

3. Tilquin P, Barrow PA, Marly J, Pitel F, Plisson-Petit F, Velge P, et al. A genome scan for quantitative trait loci affecting the Salmonella carrier-state in the chicken. Genet Sel Evol. 2005:37:539-61.

4. Calenge F, Lecerf F, Demars J, Feve K, Vignoles F, Pitel F, et al. QTL for resistance to Salmonella carrier state confirmed in both experimental and commercial chicken lines. Anim Genet. 2009;40:590-7.

5. Calenge F, Vignal A, Demars J, Fève K, Menanteau P, Velge P, et al. New QTL for resistance to Salmonella carrier-state identified on fowl microchromosomes. Mol Genet Genomics. 2011;1(285):237-43.

6. Van De GM, Blottière HM, Doré J. Humans as holobionts: implications for prevention and therapy. Microbiome. 2018;6:4-9.

7. Hanning I, Diaz-sanchez S. The functionality of the gastrointestinal microbiome in non-human animals. Microbiome. 2015:3:1-11.

8. Broom LJ, Kogut MH. The role of the gut microbiome in shaping the immune system of chickens. Vet Immunol Immunopathol. 2018:204:44-51.

9. Nurmi E, Rantala M. New aspects of Salmonella infection in broiler production. Nature. 1973:241:210-1.

10. Oakley BB, Lillehoj HS, Kogut MH, Kim WK, Maurer JJ, Pedroso A, et al. The chicken gastrointestinal microbiome. FEMS Microbiol Lett. 2014;360:100-12.

11. Clavijo V, Florez MJV. The gastrointestinal microbiome and its association with the control of pathogens in broiler chicken production: a review. Poult Sci. 2018:97:1006-21.

12. Kempf F, Menanteau P, Rychlik I, Kubasová T, Trotereau J, VirlogeuxPayant I, et al. Gut microbiota composition before infection determines the Salmonella super- and low-shedder phenotypes in chicken. Microb Biotechnol. 2020;13:1611-30.

13. Mead GC. Prospects for 'competitive exclusion' treatment to control salmonellas and other foodborne pathogens in poultry. Vet J. 2000:159:111-23.

14. Nakamura A, Ota Y, Mizukami A, Ito T, Ngwai YB, Adachi Y. Evaluation of aviguard, a commercial competitive exclusion product for efficacy and after-effect on the antibody response of chicks to Salmonella. Poult Sci. 2002;81:1653-60.

15. Sterzo E, Paiva JB, Mesquita AL, Freitas NOC, Berchieri A. Organic acids and/or compound with defined microorganisms to control Salmonella enterica serovar Enteritidis experimental infection in chickens. Braz J Poult Sci. 2007;9:69-73

16. Smialek M, Kaczorek E, Szczucińska E, Burchardt S, Kowalczyk J, Tykałowski B, et al. Evaluation of Lactobacillus spp. and yeast based probiotic (Lavipan) supplementation for the reduction of Salmonella Enteritidis after infection of broiler chickens. Pol J Vet Sci. 2019;22:5-10.

17. Varmuzova K, Kubasova T, Davidova-Gerzova L, Sisak F, Havlickova H, Sebkova A, et al. Composition of gut microbiota influences resistance of newly hatched chickens to Salmonella Enteritidis infection. Front Microbiol. 2016;7:957.

18. Hughes R-A, Ali RA, Mendoza MA, Hassan HM, Koci MD. Impact of dietary galacto-oligosaccharide (GOS) on chicken's gut microbiota, mucosal gene expression, and salmonella colonization. Front Vet Sci. 2017:4:192

19. Adhikari P, Lee CH, Cosby DE, Cox NA, Kim WK. Effect of probiotics on fecal excretion, colonization in internal organs and immune gene expression in the ileum of laying hens challenged with Salmonella Enteritidis. Poult Sci. 2019;98:1235-42.

20. Laptev GY, Filippova VA, Kochish II, Yildirim EA, Ilina LA, Dubrovin AV, et al. Examination of the expression of immunity genes and bacterial profiles in the caecum of growing chickens infected with Salmonella Enteritidis and fed a phytobiotic. Animals (Basel). 2019;9:615.

21. Kogut $\mathrm{MH}$. The effect of microbiome modulation on the intestinal health of poultry. Anim Feed Sci Technol. 2019;250:32-40.

22. Argüello H, Estellé J, Leonard FC, Crispie F, Cotter PD, O'Sullivan O, et al. Influence of the intestinal microbiota on colonization resistance to Salmonella and the shedding pattern of naturally exposed pigs. mSystems. 2019;4:e00021-19.

23. Zeng J, Lei $C$, Wang Y, Chen Y, Zhang X, Kang Z, et al. Distribution of Salmonella Enteritidis in internal organs and variation of cecum microbiota in chicken after oral challenge. Microb Pathog. 2018;122:174-9.

24. Mignon-Grasteau S, Narcy A, Rideau N, Chantry-Darmon C, Boscher $M-Y$, Sellier N, et al. Impact of selection for digestive efficiency on microbiota composition in the chicken. PLoS One. 2015;10:e0135488.

25. Ji J, Luo CL, Zou X, Lv XH, Xu YB, Shu DM, et al. Association of host genetics with intestinal microbial relevant to body weight in a chicken F2 resource population. Poult Sci. 2019;98:4084-93.

26. Birkl P, Bharwani A, Kjaer JB, Kunze W, McBride P, Forsythe P, et al. Differences in cecal microbiome of selected high and low feather-pecking laying hens. Poult Sci. 2018;97:3009-14.

27. Massacci FR, Berri M, Lemonnier G, Guettier E, Blanc F, Jardet D, et al. Late weaning is associated with increased microbial diversity and Faecalibacterium prausnitzii abundance in the fecal microbiota of piglets. Anim Microbiome. 2020;2:2.

28. Daum LT, Barnes WJ, McAvin JC, Neidert MS, Cooper LA, Huff WB, et al. Real-time PCR detection of salmonella in suspect foods from a gastroenteritis outbreak in Kerr County, Texas. J Clin Microbiol. 2002;40:3050-2.

29. Escudié F, Auer L, Bernard M, Mariadassou M, Cauquil L, Vidal K, et al. FROGS: find, rapidly, OTUs with galaxy solution. Bioinformatics. 2018:34:1287-94.

30. Magoč T, Salzberg SL. FLASH: fast length adjustment of short reads to improve genome assemblies. Bioinformatics. 2011;27:2957-63.

31. Mahé F, Rognes T, Quince C, de Vargas C, Dunthorn M. Swarm: robust and fast clustering method for amplicon-based studies. PeerJ. 2014;2:e593.

32. Rognes T, Flouri T, Nichols B, Quince C, Mahé F. VSEARCH: a versatile open source tool for metagenomics. PeerJ. 2016;4:e2584.

33. Bokulich NA, Subramanian S, Faith JJ, Gevers D, Gordon Jl, Knight R, et al. Quality-filtering vastly improves diversity estimates from Illumina amplicon sequencing. Nat Methods. 2013;10:57-9.

34. Camacho C, Coulouris G, Avagyan V, Ma N, Papadopoulos J, Bealer K, et al. BLAST+: architecture and applications. BMC Bioinformatics. 2009;10:421.

35. Caspi R, Billington R, Fulcher CA, Keseler IM, Kothari A, Krummenacker $M$, et al. The MetaCyc database of metabolic pathways and enzymes. Nucleic Acids Res. 2018;46:D633-9. 
36. Stanley D, Geier MS, Hughes RJ, Denman SE, Moore RJ. Highly variable microbiota development in the chicken gastrointestinal tract. PLoS One. 2013;8:e84290

37. Rubio LA. Possibilities of early life programming in broiler chickens via intestinal microbiota modulation. Poult Sci. 2019;98:695-706.

38. Kaiser MG, Lamont SJ. Genetic line differences in survival and pathogen load in young layer chicks after Salmonella enterica serovar enteritidis exposure. Poult Sci. 2001;80:1105-8.

39. Schou TW, Labouriau R, Permin A, Christensen JP, Sørensen P, Cu HP, et al. MHC haplotype and susceptibility to experimental infections (Salmonella Enteritidis, Pasteurella multocida or Ascaridia galli) in a commercial and an indigenous chicken breed. Vet Immunol Immunopathol. 2010;135:52-63.

40. Gast RK, Regmi P, Guraya R, Jones DR, Anderson KE, Karcher DM. Colonization of internal organs by Salmonella Enteritidis in experimentally infected laying hens of four commercial genetic lines in conventional cages and enriched colony housing. Poult Sci. 2019;98:1785-90.

41. Girard-Santosuosso O, Lantier F, Lantier I, Bumstead N, Elsen J-M, Beaumont C. Heritability of susceptibility to Salmonella enteritidis infection in fowls and test of the role of the chromosome carrying the NRAMP1 gene. Genet Sel Evol. 2002;34:211-9.

42. Lamont SJ, Kaiser MG, Liu W. Candidate genes for resistance to Salmonella enteritidis colonization in chickens as detected in a novel genetic cross. Vet Immunol Immunopathol. 2002;87:423-8.

43. Beaumont C, Protais J, Pitel F, Leveque G, Malo D, Lantier F, et al. Effect of two candidate genes on the Salmonella carrier state in fowl. Poult Sci. 2003:82:721-6.

44. Chaussé A-M, Grépinet $O$, Bottreau E, Robert V, Hennequet-Antier C, Lalmanach A-C, et al. Susceptibility to Salmonella carrier-state: a possible Th2 response in susceptible chicks. Vet Immunol Immunopathol. 2014;159:16-28.

45. Videnska P, Sisak F, Havlickova H, Faldynova M, Rychlik I. Influence of Salmonella enterica serovar Enteritidis infection on the composition of chicken cecal microbiota. BMC Vet Res. 2013;9:140.

46. Juricova H, Videnska P, Lukac M, Faldynova M, Babak V, Havlickova H, et al. Influence of Salmonella enterica serovar enteritidis infection on the development of the cecum microbiota in newly hatched chicks. Appl Environ Microbiol. 2013;79:745-7.

47. Liu L, Lin L, Zheng L, Tang H, Fan X, Xue N, et al. Cecal microbiome profile altered by Salmonella enterica, serovar Enteritidis inoculation in chicken. Gut Pathog. 2018;10:34

48. Mon KKZ, Saelao P, Halstead MM, Chanthavixay G, Chang H-C, Garas L, et al. Salmonella enterica serovars Enteritidis infection alters the indigenous microbiota diversity in young layer chicks. Front Vet Sci. 2015;2:61.

49. Chintoan-Uta C, Wisedchanwet T, Glendinning L, Bremner A, Psifidi A, Vervelde $L$, et al. Role of cecal microbiota in the differential resistance of inbred chicken lines to colonization by Campylobacter jejuni. Appl Environ Microbiol. 2020;86:e02607-e2619.

50. Goodrich JK, Davenport ER, Waters JL, Clark AG, Ley RE. Cross-species comparisons of host genetic associations with the microbiome. Science. 2016;352:532-5.

51. Schokker D, Veninga G, Vastenhouw SA, Bossers A, de Bree FM, KaalLansbergen LMTE, et al. Early life microbial colonization of the gut and intestinal development differ between genetically divergent broiler lines. BMC Genomics. 2015;16:418.

52. Zhao L, Wang G, Siegel P, He C, Wang H, Zhao W, et al. Quantitative genetic background of the host influences gut microbiomes in chickens. Sci Rep. 2013;3:1163.

53. Pandit RJ, Hinsu AT, Patel NV, Koringa PG, Jakhesara SJ, Thakkar JR, et al. Microbial diversity and community composition of caecal microbiota in commercial and indigenous Indian chickens determined using 16s rDNA amplicon sequencing. Microbiome. 2018;6:115.

54. Meng H, Zhang Y, Zhao L, Zhao W, He C, Honaker CF, et al. Body weight selection affects quantitative genetic correlated responses in gut microbiota. PLoS One. 2014;9:e89862.

55. Mon KKZ, Zhu Y, Chanthavixay G, Kern C, Zhou H. Integrative analysis of gut microbiome and metabolites revealed novel mechanisms of intestinal Salmonella carriage in chicken. Sci Rep. 2020;10:4809.

56. Goodrich JK, Waters JL, Poole AC, Sutter JL, Koren O, Blekhman R, et al. Human genetics shape the gut microbiome. Cell. 2014;159:789-99.

57. Waters JL, Ley RE. The human gut bacteria Christensenellaceae are widespread, heritable, and associated with health. BMC Biol. 2019;17:83.
58. Biagi E, Franceschi C, Rampelli S, Severgnini M, Ostan R, Turroni S, et al. Gut microbiota and extreme longevity. Curr Biol. 2016;26:1480-5.

59. Stenman LK, Burcelin R, Lahtinen S. Establishing a causal link between gut microbes, body weight gain and glucose metabolism in humanstowards treatment with probiotics. Benef Microbes. 2016;7:11-22.

60. Litvak Y, Mon KKZ, Nguyen H, Chanthavixay G, Liou M, Velazquez EM, et al. Commensal Enterobacteriaceae protect against Salmonella colonization through oxygen competition. Cell Host Microbe. 2019;25:128-139.e5.

61. Deriu E, Liu JZ, Pezeshki M, Edwards RA, Ochoa RJ, Contreras H, et al. Probiotic bacteria reduce Salmonella typhimurium intestinal colonization by competing for iron. Cell Host Microbe. 2013;14:26-37.

62. Rychlik I. Composition and function of chicken gut microbiota. Animals (Basel). 2020;10:103.

63. Winter SE, Thiennimitr P, Winter MG, Butler BP, Huseby DL, Crawford RW, et al. Gut inflammation provides a respiratory electron acceptor for Salmonella. Nature. 2010;467:426-9.

64. Parada Venegas D, De la Fuente MK, Landskron G, González MJ, Quera R, Dijkstra G, et al. Short chain fatty acids (SCFAs)-mediated gut epithelial and immune regulation and its relevance for inflammatory bowel diseases. Front Immunol. 2019;10:277.

65. Schulthess J, Pandey S, Capitani M, Rue-Albrecht KC, Arnold I, Franchini $F$, et al. The short chain fatty acid butyrate imprints an antimicrobial program in macrophages. Immunity. 2019;50:432-445.e7.

66. Valdes AM, Walter J, Segal E, Spector TD. Role of the gut microbiota in nutrition and health. BMJ. 2018;361:k2179.

67. Zou X, Ji J, Qu H, Wang J, Shu DM, Wang Y, et al. Effects of sodium butyrate on intestinal health and gut microbiota composition during intestinal inflammation progression in broilers. Poult Sci. 2019:98:4449-56

68. Van Immerseel F, De Buck J, Pasmans F, Velge P, Bottreau E, Fievez V, et al. Invasion of Salmonella enteritidis in avian intestinal epithelial cells in vitro is influenced by short-chain fatty acids. Int J Food Microbiol. 2003:85:237-48.

69. Hiller CC, Lucca V, Carvalho D, Borsoi A, Borges KA, Furian TQ, et al. Influence of catecholamines on biofilm formation by Salmonella Enteritidis. Microb Pathog. 2019;130:54-8.

70. Lyte M, Vulchanova L, Brown DR. Stress at the intestinal surface: catecholamines and mucosa-bacteria interactions. Cell Tissue Res. 2011;343:23-32.

71. Methner U, Rabsch W, Reissbrodt R, Williams PH. Effect of norepinephrine on colonisation and systemic spread of Salmonella enterica in infected animals: role of catecholate siderophore precursors and degradation products. Int J Med Microbiol. 2008;298:429-39.

72. Pullinger GD, van Diemen PM, Carnell SC, Davies H, Lyte M, Stevens MP. 6-hydroxydopamine-mediated release of norepinephrine increases faecal excretion of Salmonella enterica serovar Typhimurium in pigs. Vet Res. 2010;41:68.

73. Boyen F, Eeckhaut V, Van Immerseel F, Pasmans F, Ducatelle R, Haesebrouck F. Quorum sensing in veterinary pathogens: mechanisms, clinical importance and future perspectives. Vet Microbiol. 2009;135:187-95.

74. Schonewille E, Nesse LL, Hauck R, Windhorst D, Hafez HM, Vestby LK. Biofilm building capacity of Salmonella enterica strains from the poultry farm environment. FEMS Immunol Med Microbiol. 2012;65:360-5.

75. Polansky O, Sekelova Z, Faldynova M, Sebkova A, Sisak F, Rychlik I. Important metabolic pathways and biological processes expressed by chicken cecal microbiota. Appl Environ Microbiol. 2015;82:1569-76.

76. Xi Y, Shuling N, Kunyuan T, Qiuyang Z, Hewen D, ChenCheng G, et al. Characteristics of the intestinal flora of specific pathogen free chickens with age. Microb Pathog. 2019;132:325-34.

\section{Publisher's Note}

Springer Nature remains neutral with regard to jurisdictional claims in published maps and institutional affiliations. 